\title{
ANÁLISIS DE LAS DIFERENCIAS CULTURALES EN EMPRESAS MÉXICO-ALEMANAS A TRAVÉS DEL MODELO DE HOFSTEDE
}

\author{
Cultural differences in Mexican-German companies analyzed using Hofstede's model
}

\author{
Franziska Tengler \\ Licenciatura en Ciencias Empresariales con enfoque en Marketing, Universidad de Guanajuato, \\ Guanajuato - México, franzite@online.de \\ Diana del Consuelo Caldera-González \\ Doctorado en Estudios Organizacionales, Universidad de Guanajuato, Guanajuato- México, \\ dccaldera@ugto.mx
}

\begin{abstract}
Cómo referenciar / How to cite
Tengler, F. y Caldera-Gónzalez, D. (2018). Análisis de las diferencias culturales en empresas México-alemanas a través del modelo de Hofstede. Revista CEA, 4(7), 49-65. https://doi.org/10.22430/24223182.759
\end{abstract}

Recibido: 31 de julio de 2017

Aceptado: 30 de septiembre de 2017

\section{Resumen}

Actualmente, las relaciones comerciales entre México y Alemania se han fortalecido, siendo México el único país latinoamericano con un Tratado de Libre Comercio con Alemania.

El objetivo de este trabajo es analizar el modelo cultural de Hofstede, para explicar las similitudes y diferencias culturales entre organizaciones alemanas y mexicanas, lo cual permite generar una gestión exitosa del talento humano en un entorno multicultural.

Se utilizó el modelo cultural de Geert Hofstede por ser uno de los enfoques culturales más reconocidos acerca de la investigación intercultural.

Se trata de una investigación documental con enfoque descriptivo, cuyas conclusiones resaltan que la cultura alemana y la cultura mexicana coinciden solamente en dos de las dimensiones propuestas por Hofstede: la masculinidad y la evitación de incertidumbre. Además, se encontró que Alemania tiene una distancia de poder pequeña y una orientación a largo plazo; por su parte México posee una distancia de poder alta y una orientación a corto plazo. Más allá, el individualismo y la restricción prevalecen en organizaciones alemanas, mientras que el colectivismo y la indulgencia sobresalen en empresas mexicanas.

Indiscutiblemente, las diferencias mencionadas deben considerarse cuando se desea una gestión exitosa del talento humano en el entorno mexicano-alemán.

Palabras clave: multiculturalidad, liderazgo, capital humano, México, Alemania.

\begin{abstract}
Current commercial relationships between Mexico and Germany are stronger because said Latin American country is the only case of a
\end{abstract}


Free Trade Agreement with that European counterpart. The objective of this work is to analyze Geert Hofstede's cultural model and explain the similarities and differences between Mexican and German organizations, which enables to successfully manage human talent in multicultural environments. Such model was adopted as it is one of the most widely-known cultural approaches in intercultural research, and this documentary study took a descriptive approach. The conclusions highlight the fact that these cultures only coincide in two of the dimensions proposed by Hofstede: masculinity and uncertainty avoidance. Besides, Germany was found to have short power distance and longterm orientation. In turn, Mexico presented a long power distance and short-term orientation. Furthermore, individualism and restriction dominate German organizations, while collectivism and indulgence stand out in Mexican companies. These differences must be considered when successful human talent management in the Mexican-German context is the goal.

Keywords: multiculturality, leadership, human talent, Mexico, Germany.

\section{INTRODUCCIÓN}

Hoy en día la globalización representa un fenómeno común con efectos en la economía, la política y la sociedad. Dependiendo del sistema económico de cada país, la globalización puede ser considerada como una amenaza o como una oportunidad. Bajo el aspecto de que ningún país es autosuficiente, necesariamente existen relaciones internacionales las cuales aumentan cada vez más en el contexto de la globalización.

Para colaborar exitosamente en un ambiente profesional, es indispensable entender las dimensiones de una organización internacional, ya que en la interacción entre empresas internacionales pueden surgir situaciones complejas, debido a culturas y costumbres diferentes (Garza, Guzmán \& Hernández, 2009).

Según Barkley y Eggertsson (2017), una estrategia de liderazgo para las empresas que operan a través de fronteras nacionales es el fundamento para mejorar su rendimiento económico. Así, a través de una estrategia de gestión efectiva, es posible: a) sensibilizar respecto a culturas distintas; b) que los empleados se adapten más fácilmente; c) que exista una comunicación eficaz entre gerentes y subordinados; y d) alinear los incentivos y los objetivos.

El modelo cultural de Geert Hofstede ayuda a entender distintas culturas y, en consecuencia, el comportamiento de los involucrados en una empresa, a partir de esto, dicho modelo permite desarrollar una estrategia de gestión multicultural. Si se consideran las necesidades de todos los empleados, los gerentes pueden propiciar un ambiente favorable, lo cual, a su vez, genera un mejor desempeño (Barkley \& Eggertsson, 2017).

Considerando que la cultura influye en gran medida en el estilo de liderazgo preferido por los empleados, y este a su vez determina su desempeño, el objetivo del presente trabajo es analizar el modelo cultural de Hofstede, para explicar las similitudes y diferencias culturales entre organizaciones alemanas y mexicanas. Este trabajo sirve como punto de partida para una futura investigación acerca de la percepción y la autopercepción del estilo de liderazgo en el ámbito profesional mexicanoalemán, ya que ambos países han reforzado sus relaciones económicas en los últimos años.

Alemania es el socio comercial más importante de México en la Unión Europea, y en el mundo ocupa el quinto lugar. Por su parte, para 
Alemania, México es el primer socio económico más importante en América Latina. Entre los países de la Unión Europea que invierten en México, Alemania ocupa el cuarto lugar, y en todo el mundo es el sexto inversor más importante (Peña, 2016).

Se considera relevante este estudio, puesto que las relaciones económicas entre Alemania y México han tenido un considerable crecimiento en el comercio bilateral del $60 \%$ entre 2000 y 2015, el cual fue todavía más significativo entre los años 2000 y 2015 con un aumento del 150 \% por la entrada en vigor del Tratado de Libre Comercio entre la Unión Europea y México en el cambio del milenio. El $98 \%$ de los productos más comercializados entre México y Alemania provienen de la industria automovilística, farmacéutica, maquinaria de alta precisión, electrónica y ferretería (Embajada de México en Alemania, 2016).

\section{MARCO TEÓRICO-EMPÍRICO}

La explicación del comportamiento de las personas, sea de forma individual o colectiva, es el enfoque principal de las teorías de las ciencias sociales. En el ámbito de la investigación en gestión, dichas ciencias pretenden comprender el comportamiento de los involucrados, pero situados en organizaciones concretas (McSweeney, Brown \& Iliopoulou, 2016).

La literatura de Geert Hofstede, acerca de los agrupamientos culturales por regiones y países, puede considerarse como la teoría a este respecto más influyente en el ámbito empresarial. Sus investigaciones datan desde los años sesenta (Hofstede, 1983 and 1993), inspirando diversos estudios empíricos, hasta nuestros días (Khlif, 2016; Kirkman, Lowe \& Gibson, 2006; Ng, Lee, Soutar, 2007; Venaik,
Zhu \& Brewer, 2013), motivo por el cual se considera en este trabajo.

\section{Cultura como programación mental}

De acuerdo con Hofstede, la manera como las personas piensan, sienten y actúan se entiende como una programación mental, o como el software de la mente, haciendo referencia a computadoras que son programadas. En seguida se hace uso de los términos en inglés: mental programs y software of the mind. A través del software mental se pueden entender ciertas reacciones de un individuo. Sin embargo, esto no quiere decir que el comportamiento de una persona está completamente predeterminado. Una persona siempre tiene la opción de responder de forma inesperada, independientemente de sus programas mentales.

La fuente de dichos programas se encuentra en el entorno social en el que el individuo ha crecido y ha recogido su experiencia de vida. El punto de partida para la «programación» es la familia, continuado por el vecindario, la escuela y el trabajo. Un sinónimo para el mental software es la cultura, la cual representa un fenómeno colectivo porque se comparte con otras personas que viven en el mismo entorno donde se aprendió. La cultura distingue entre miembros de distintos grupos. Así que la cultura deriva del entorno social y no de los genes, se aprende y no se hereda. La cultura se distingue de la naturaleza humana y de la personalidad del individuo. La Figura 1 muestra dicho constructo, lo cual se explica en seguida con detalle.

Como un nivel universal, la naturaleza humana representa a los requisitos y características humanas que todos los individuos poseen independientemente de su cultura. Estos incluyen el amor, la tristeza, la alegría, la culpa o la vergüenza y también la necesidad de la interacción social y comunicación, así como la 
participación comunitaria. Estos sentimientos son heredados genéticamente. La cultura determina de qué manera estas necesidades humanas y los sentimientos son expresados.

Por otra parte, la personalidad de un individuo consiste en el conjunto individual de los mental programs, los cuales se comparten con ningún otro individuo. Se basa en características que en parte se heredan por la combinación única de genes de la persona y en parte son aprendidas, lo que quiere decir que la personalidad es modificada por la influencia de la cultura y experiencias del individuo.

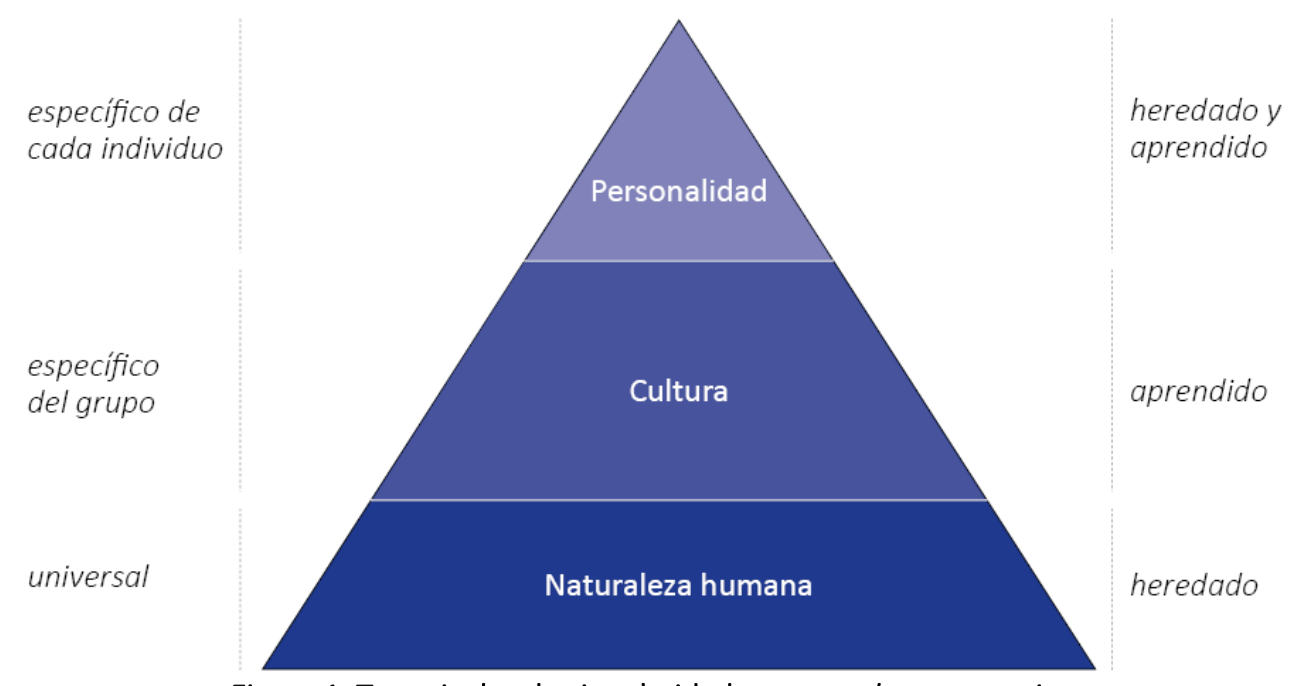

Figura 1. Tres niveles de singularidad en mental programming

Figure 1. Three singularity levels in mental programming Fuente: elaboración propia con base en Hofstede, Hofstede y Minkov, 2010.

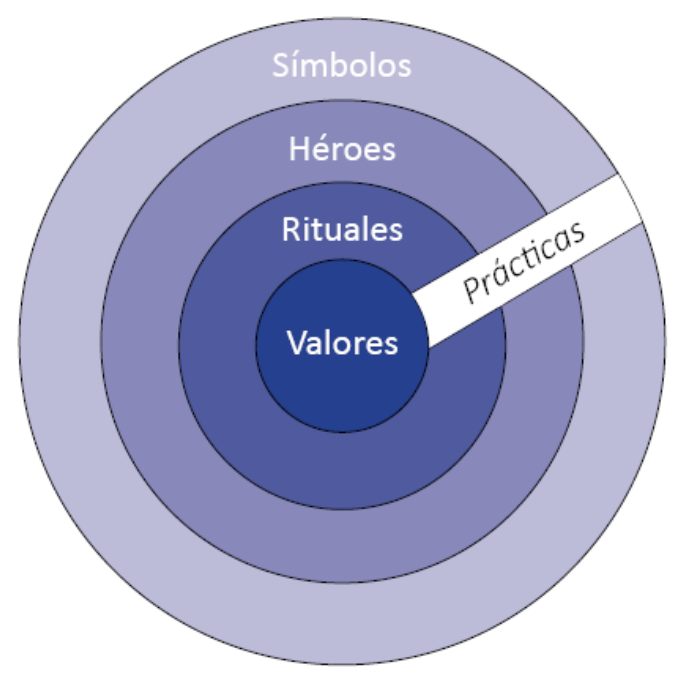

Figura 2. Manifestaciones de cultura en diferentes niveles de profundidad

Figure 2. Cultural manifestations at different levels

Fuente: elaboración propia con base en Hofstede, Hofstede y Minkov, 2010. 
Valores, rituales, héroes y símbolos

Según Hofstede, las diferencias entre culturas se muestran a través de valores, rituales, héroes y símbolos, los cuales se pueden ver en la Figura 2, donde se pueden apreciar los diferentes niveles de profundidad. Los símbolos representan la manifestación más superficial y los valores la más profunda.

Como símbolos se entienden palabras, gestos, imágenes u objetos que tienen un cierto significado para los que comparten la cultura.

Este tipo de manifestación se desarrolla continuamente, así que nuevos símbolos aparecen, mientras que otros desaparecen o se imitan de otras culturas. Por tal razón, los símbolos se encuentran en el nivel más superficial. Los héroes representan personas vivas 0 muertas las cuales poseen características altamente apreciadas y que funcionan como un modelo a seguir. Por un lado, superfluos para el alcance de objetivos, sin embargo, los rituales se consideran socialmente esenciales. Se llevan a cabo por su propio bien, como ejemplo se puede mencionar: maneras de saludar, ceremonias religiosas o sociales. No obstante que el significado cultural de las tres manifestaciones mencionadas es invisible, estas tienen en común la visibilidad para extraños, ilustrado en la Figura 2 por el término prácticas. En el centro se encuentran los valores, los cuales representan tendencias de preferencia de ciertos estados de cosas sobre otras. Los valores están reflejados en sentimientos contradictorios, como lo racional e irracional o lo moral e inmoral.

La Figura 3 muestra la adquisición de los valores y prácticas durante la vida. Mientras que los valores son adquiridos temprano $y$ principalmente por parte de la familia, las prácticas se obtienen más tarde y sobre todo en relación con el trabajo. Clarificando un poco más en una etapa de 10 a 12 años, en la cual el ser humano posee una alta capacidad de absorción, se impregnan símbolos, héroes, rituales y los valores básicos. Al final de dicho periodo, el aprendizaje cambia de tal manera, que es más consciente y se enfoca en nuevas prácticas.

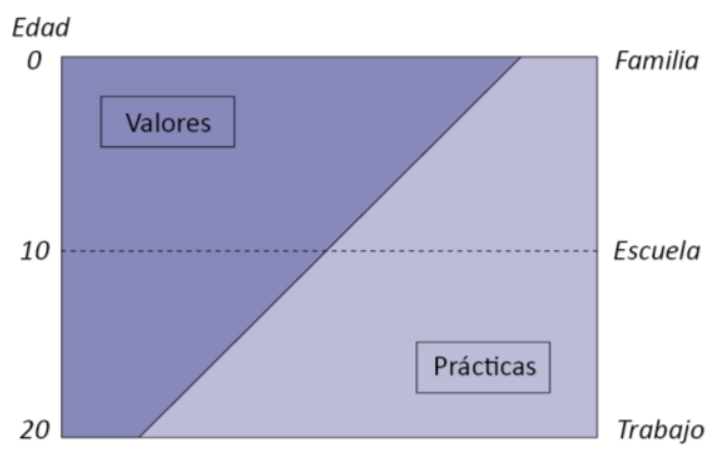

Figura 3. Aprendizaje de valores y prácticas

Figure 3. Learning values and practices

Fuente: elaboración propia con base en Hofstede, Hofstede y Minkov, 2010.

\section{Dimensiones culturales}

Para el estudio de diferencias culturales, Geert Hofstede tuvo la oportunidad de aplicar y analizar una gran cantidad de encuestas sobre los valores de los empleados de la empresa IBM en más de 70 países y regiones, entre ellos Alemania y México. A través de un procedimiento estadístico y de análisis factorial, se agruparon las preguntas y se les denominó clústeres. Se trató de un estudio cuantitativo analizando las respuestas de los encuestados a través de una escala precodificada de 1, 2, 3, 4 y 5. Las cuatro áreas que detectó Hofstede fueron las siguientes: 1) distancia de poder; 2) colectivismo versus individualismo; 3) feminidad versus masculinidad; y 4) evitación de la incertidumbre. Dichas áreas son llamadas "dimensiones culturales», cuyo término inglés es dimensions of cultures. Como dimensión se entiende un aspecto de una cultura el cual se 
puede medir en comparación con otras culturas. Las dimensiones de Hofstede coincidieron con los descubrimientos de los científicos Alex Inkeles y Daniel Levinson 20 años antes. Posteriormente al descubrimiento y análisis de las cuatro dimensiones mencionadas, Minkov desarrolló otras dos categorías que complementan el modelo de Hofstede: 5) orientación a largo plazo versus orientación a corto plazo; y 6) indulgencia versus restricción. En la Tabla 1 se describen las seis dimensiones (Hofstede, Hofstede and Minkov, 2010).

Tabla 1. Dimensiones culturales de Hofstede

Table 1. Hofstede's cultural dimensions

\begin{tabular}{|c|c|}
\hline Baja distancia de poder & Alta distancia de poder \\
\hline Dependencia limitada de los empleados y sus jefes & Dependencia alta de los empleados y sus jefes \\
\hline Baja distancia emocional entre los empleados y sus jefes & Alta distancia emocional entre los empleados y sus jefes \\
\hline Los subordinados se acercan y contradicen fácilmente con & Los subordinados no contactan o contradicen sus líderes \\
\hline sus jefes & de manera directa \\
\hline Los seguidores y líderes son considerados iguales & Los seguidores y líderes son considerados desiguales \\
\hline Organización descentralizada & Organización centralizada \\
\hline Niveles jerárquicos planos & Muchos niveles jerárquicos \\
\hline Personal de supervisores limitado & Mucho personal de supervisores \\
\hline Rangos de salario equilibrados & Rangos de salario desequilibrados \\
\hline Empleados extremamente cualificados y privilegiados & Empleados con bajos niveles educativos \\
\hline Líderes jóvenes más apreciados & Líderes autócratas y paternos \\
\hline Individualismo & Colectivismo \\
\hline Empleados actúan según sus propios intereses & Empleados actúan según los intereses del grupo \\
\hline Evitación de empleo de parientes del personal & Empleo de parientes del personal \\
\hline $\begin{array}{l}\text { Relación entre superiores e inferiores como una } \\
\text { transacción de negocios calculable }\end{array}$ & $\begin{array}{l}\text { Relación entre superiores e inferiores como estructuras } \\
\text { familiares }\end{array}$ \\
\hline Rendimiento bajo o una oferta mejor justifican la & Rendimiento bajo de un empleado no justifica un despido \\
\hline terminación de una relación profesional & Administración enfocada en el grupo \\
\hline Administración enfocada en el rendimiento del individuo & Emisión de incentivos y bonificaciones a todo un grupo \\
\hline \multicolumn{2}{|l|}{$\begin{array}{l}\text { Emisión de incentivos individuales, recompensas y } \\
\text { bonificaciones }\end{array}$} \\
\hline Masculinidad & Feminidad \\
\hline $\begin{array}{l}\text { Resolución de problemas a través de luchas, de tal } \\
\text { manera que el mejor gana }\end{array}$ & $\begin{array}{l}\text { Resolución de problemas a través de negociación y } \\
\text { compromiso }\end{array}$ \\
\hline Recompensa del desempeño con base en la equidad & Recompensa del desempeño con base en la igualdad \\
\hline Asignación de una prioridad alta al trabajo & Asignación de una prioridad baja al trabajo \\
\hline Apreciación del salario más que el tiempo libre & Apreciación del tiempo libre más que el salario \\
\hline Valores esenciales: asertividad, ambición, competencia & Valores esenciales: modestia, solidaridad \\
\hline La mayoría de las mujeres jóvenes no pretenden una & Los dos géneros pretenden una carrera \\
\hline carrera & Organizaciones pequeñas \\
\hline Organizaciones grandes & Servicios, fabricación según las necesidades de los \\
\hline $\begin{array}{l}\text { Fabricación y producción de equipo pesado y en } \\
\text { volúmenes grandes }\end{array}$ & clientes, manejo de materia viva \\
\hline
\end{tabular}




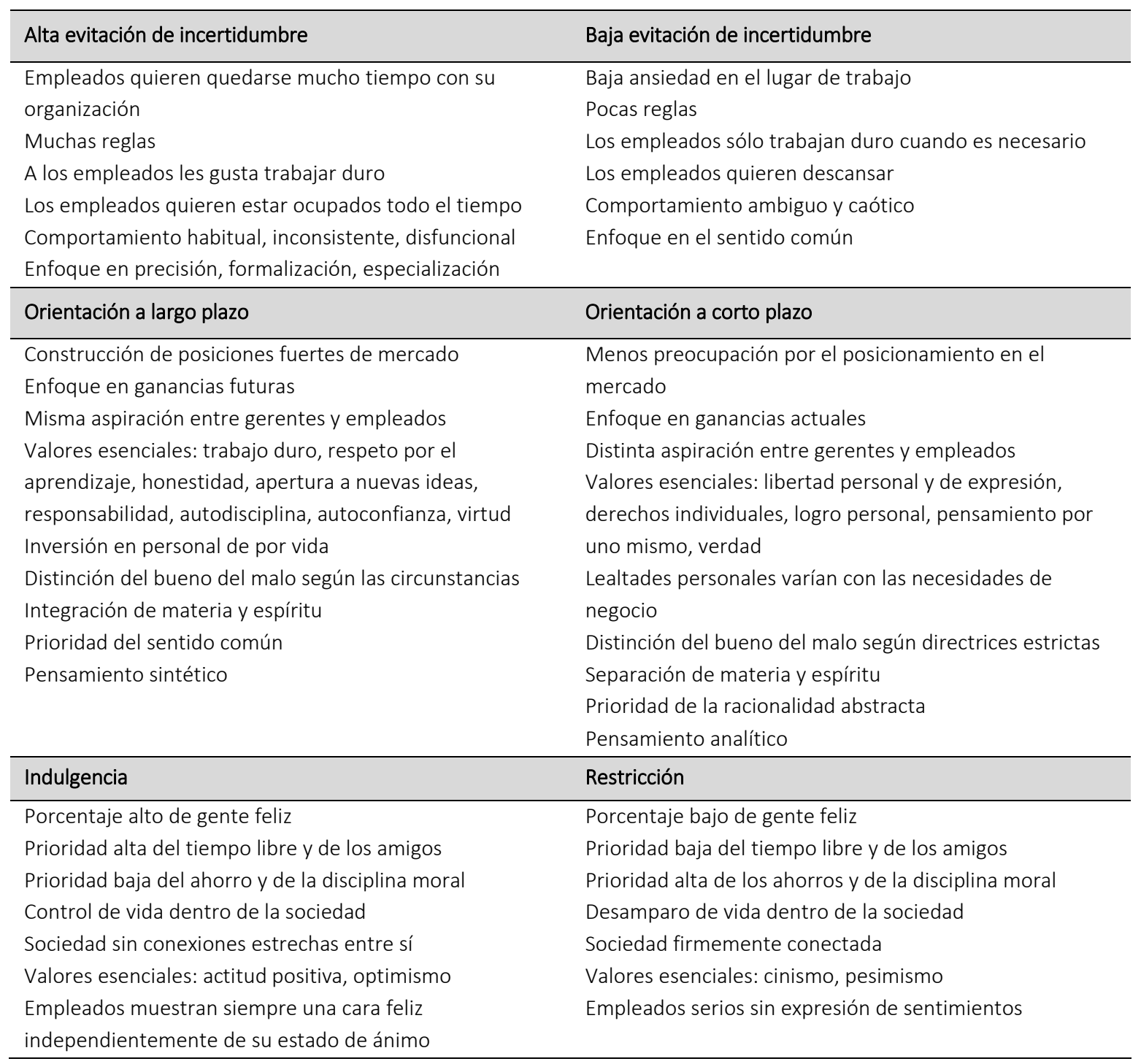

Fuente: elaboración propia con base en Hofstede y Minkov, 2010.

\section{METODOLOGÍA}

Para lograr el objetivo del análisis del modelo cultural de Geert Hofstede para explicar las similitudes y diferencias entre el comportamiento de las organizaciones bajo la influencia de grupos culturales nacionales de Alemania y México, se realizó una investigación de corte no experimental basada en análisis documental de fuentes primarias y secundarias de información, todo con un alcance exploratorio.
Inicialmente se analizó el término 'cultura' según Hofstede, con sus símbolos, héroes, rituales y valores para entender este concepto, basándonos en los estudios realizados por este autor. Posteriormente, se estudiaron las cuatro dimensiones del estudio IBM de Hofstede, así como las dos dimensiones del World Value Survey (WVS, representa un estudio con enfoque en los elementos de la cultura nacional con sus valores y normas. Se considera la encuesta más extensa acerca de los valores humanos.) de Minkov que complementan el modelo de Hofstede, para comprender la 
influencia de la cultura en especial en los empleados, así como en la estructura de las organizaciones.

Por último, se estudiaron los índices de Alemania y México obtenidos en cada una de las dimensiones culturales y se vincularon los resultados de un país con el otro para obtener una comparación de las similitudes y diferencias en cuanto al comportamiento de la gente dentro de las organizaciones alemanas y mexicanas, realizándose un análisis comprensivo de los estudios de Geert Hofstede.

Con referencia al diseño del presente trabajo, este corresponde a un diseño no experimental, ya que es totalmente teórico, no obstante, se considera como punto de partida para realizar una investigación aplicada para estudiar el liderazgo en el contexto mexicano-alemán.

\section{RESULTADOS Y DISCUSIÓN}

El estudio de Hofstede se desarrolló a finales de los años sesenta del siglo XX, cuando Hofstede empezó con el análisis de los datos de un amplio ejercicio de encuestas de personal de la empresa IBM en 76 países (Hofstede, 1984).

La primera dimensión que se analizó fue la de distancia de poder. México obtuvo el 10 y 11 lugar con un índice de 81, y Alemania del 65 al 67 lugar con un índice de 35, de 76 países en total. Mientras que México muestra valores altos en esta dimensión, Alemania muestra valores bajos.

Esta dimensión tiene un gran impacto en cuanto a la planificación y el control de las organizaciones, así que el control representa una forma de poder. Un índice alto en la dimensión de distancia de poder, como lo tiene México, implica un pensamiento más político que estratégico, como lo es en el caso de Alemania. Por otra parte, culturas con un índice alto apoyan la planificación personal y el control, mientras que países como Alemania apoyan los sistemas impersonales. Un índice alto, así como una posición alta en la jerarquía significa una planificación y control informales. En países con un índice bajo, como en el caso de Alemania, los sistemas de control confían más en los seguidores; mientras que en países con índices altos no existe confianza, sin embargo, la dependencia de personas que ostentan poder es una necesidad básica la cual puede ser un factor de motivación. Lo que motiva a las personas en las culturas como la de Alemania, con un índice bajo, son reglas reforzadas por un superego. No obstante, la gestión participativa es un medio valioso en dichas culturas, mientras que, en países como México, la distribución del poder es una contradicción, la cual puede resultar en resistencia por parte de los superiores, así como de los subordinados. Los países con una distancia de poder alta no surgen del desarrollo sino a través de crisis o revoluciones, ya que los procesos de desarrollo generan incertidumbre en los individuos.

También la dimensión de evitación de incertidumbre tiene influencia en la planificación y el control, por el hecho de que la planificación es una forma de reducir la incertidumbre. En dicha dimensión, México se encuentra en los lugares 26 y 27 con un índice de 82; Alemania está entre el 43 y 44 lugar, con un índice de 65 entre un total de 76 países participantes. Los dos países se encuentran en medio del rango, con una tendencia hacia la evitación de la incertidumbre. 


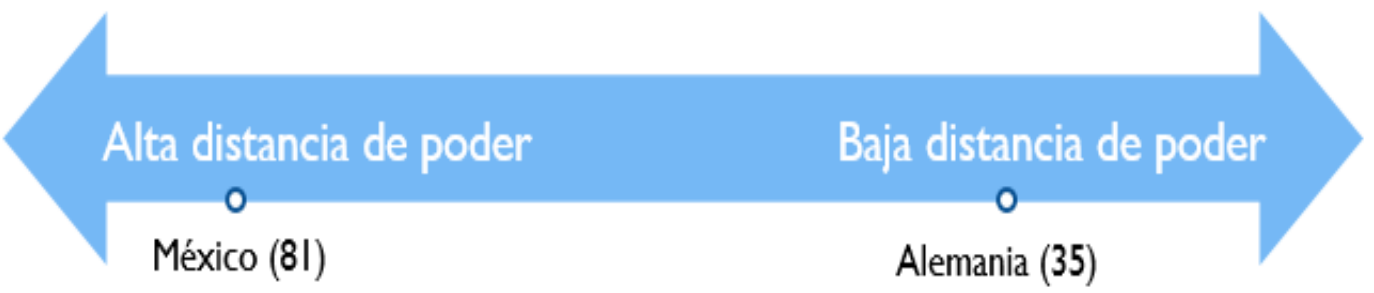

Figura 4. Puntuación de México y Alemania en la dimensión de distancia de poder

Figure 4. Power distance scores of Mexico and Germany Fuente: elaboración propia.

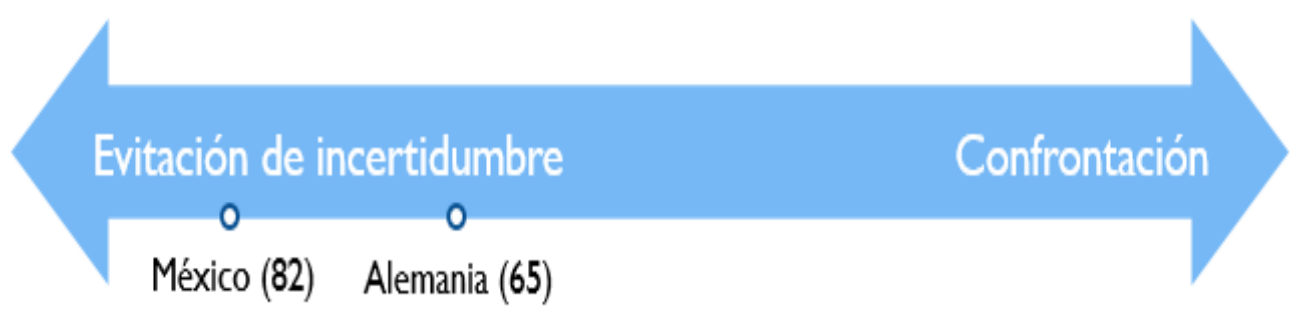

Figura 5. Puntuación de México y Alemania en la dimensión de evitación de incertidumbre Figure 5. Uncertainty avoidance scores of Mexico and Germany

Fuente: elaboración propia.

Para recabar un alto índice en la dimensión de evitación de incertidumbre, significa que países como México y Alemania evitan una planificación estratégica, porque esta puede generar incertidumbre. De esta manera, culturas con una alta evitación a la incertidumbre tienen la necesidad de una planificación en detalle, la cual, en la mayoría de los casos, es responsabilidad de los especialistas y de una retroalimentación a corto plazo.

Las dos dimensiones, distancia de poder y evitación de incertidumbre, se consideran como puntos clave para la estructura de una organización. Estudios comprueban que organizaciones que se establecen en el extranjero continúan ejerciendo el sistema de planificación y control que tienen en su país de origen. De aquí pueden surgir problemas, ya que la otra cultura puede mostrar otros valores y costumbres. En este caso particular, México muestra una distancia de poder grande en contraste con Alemania, por lo cual empleados mexicanos prefieren tener una distancia grande con sus líderes, no los confrontan ni les contradicen. Por el contrario, los empleados alemanes mantienen una distancia pequeña con sus superiores y por lo tanto se acercan más con sus jefes y les contradicen más fácilmente. Las diferentes costumbres y valores que surgen deben respetarse y de los cuales los gerentes tienen que estar conscientes para poder conciliarlos de manera adecuada.

En cuanto a la dimensión de colectivismo versus individualismo, se puede observar una gran brecha entre Alemania en el 19 lugar con un índice de 67 y México en el 48 y 50 lugar con un índice de 30, de un total de 76 países. Esto significa que mientras que en México prevale el colectivismo, en Alemania domina el individualismo. 


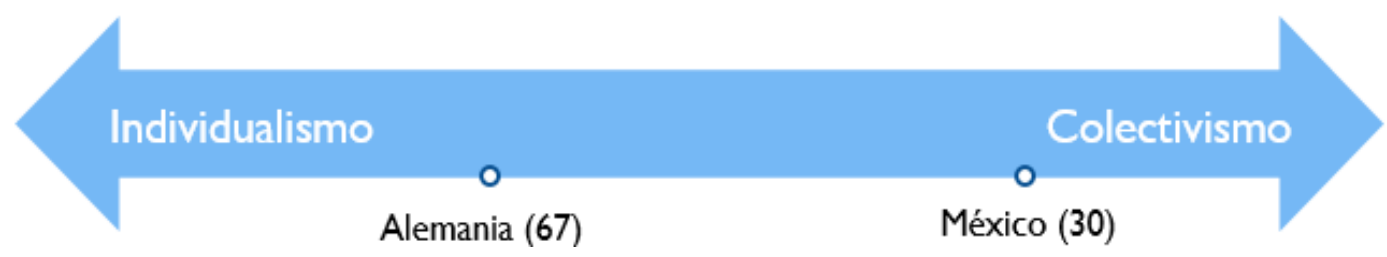

Figura 6. Puntuación de México y Alemania en la dimensión de colectivismo versus individualismo

Figure 6. Collectivism vs. individualism scores of Mexico and Germany

Fuente: elaboración propia..

El análisis logístico binario se desarrolló en el programa SPSS, mediante el método «por pasos hacia adelante» con el criterio de Wald; en el modelo se van añadiendo variables independientes, según su grado de relación con la variable dependiente y su significación estadística. En la Tabla 2 aparecen 107 casos introducidos, que son los seleccionados para el análisis y los casos excluidos, en este caso son cero. En cuanto a la planificación y el control, en países como Alemania, con una cultura de individualismo, la evaluación de desempeño es indispensable, en cambio en países con un índice bajo no se toma con igual de seriedad. Culturas como México tienen otros métodos para definir el rendimiento de las organizaciones y de los empleados. La evaluación de desempeño se queda sin gran impacto. En países donde predomina el colectivismo, la relación entre los empleados y la organización se basa en la moral, mientras que en países como Alemania donde predomina el individualismo, esta relación se enfoca más en los intereses propios. Resulta evidente que aquí se enfrentan dos enfoques diferentes los cuales pueden ocasionar conflictos si no se consideran estas diferencias culturales.

Al igual que en la dimensión de evitación de incertidumbre, los índices de México y
Alemania en la dimensión de feminidad versus masculinidad son similares. México está en el 8 lugar con un índice de 69 y Alemania en el 11 y 13 lugar, con un índice de 66, donde representa a culturas femeninas y 100 a culturas masculinas.

Así es que las culturas de los dos países se pueden considerar como masculinas. La diferencia entre los índices de México y Alemania es muy pequeña. Este hecho puede favorecer la colaboración, de tal manera que los empleados sigan los mismos valores. Cuando se trata de la resolución de problemas, el enfoque en resultados o la recompensa según la equidad, los individuos comparten la misma comprensión. La gente de las dos culturas tiene la misma apreciación en cuanto a la alta prioridad del trabajo en la vida y el menosprecio del tiempo libre. La asertividad, la ambición y la competencia representan valores importantes en las culturas marcadas por la masculinidad.

En la dimensión de orientación a largo plazo, Alemania se encuentra en el sexto lugar con un índice de 83 y México en el 74 y 76 lugar, con un índice de 24 en un rango de 0 a 100, en donde 0 representa la orientación a corto plazo y 100 a largo plazo. 


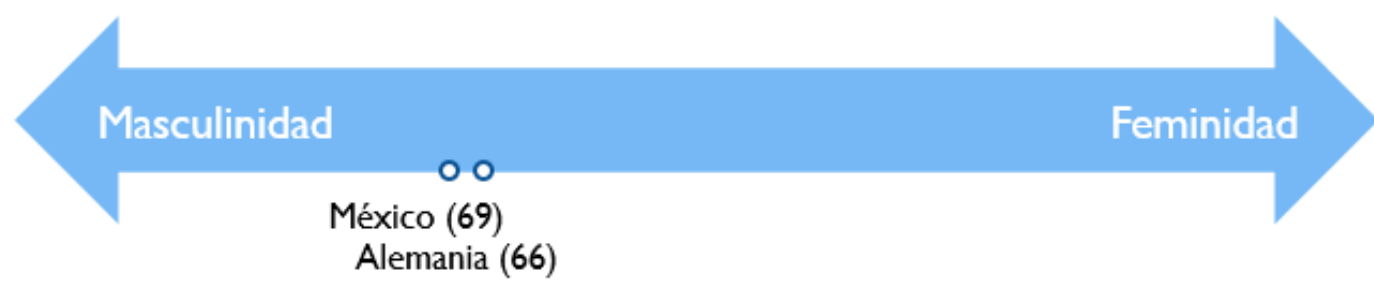

Figura 7. Puntuación de México y Alemania en la dimensión de feminidad versus masculinidad

Figure 7. Masculinity vs. femininity scores of Mexico and Germany Fuente: elaboración propia.

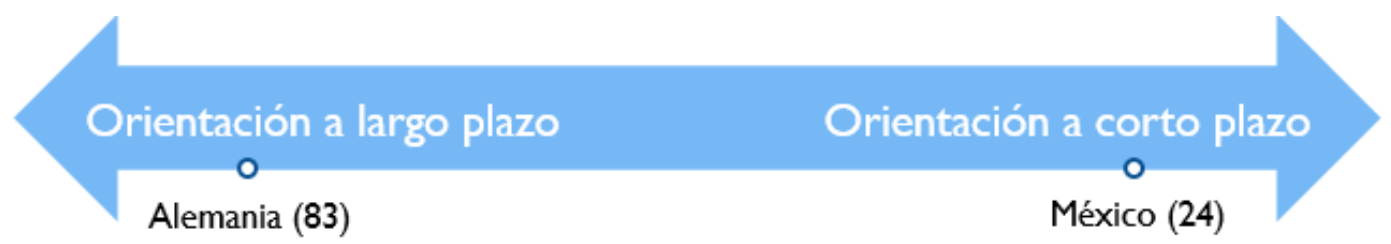

Figura 8. Puntuación de México y Alemania en la dimensión de orientación a largo plazo

Figure 8. Long-term orientation scores of Mexico and Germany Fuente: elaboración propia.

El índice de Alemania muestra que su cultura se enfoca en la orientación a largo plazo, mientras que el índice de México demuestra un enfoque en la orientación a corto plazo. De aquí pueden resultar conflictos así que los enfoques de los países son totalmente opuestos. Valores con gran importancia dentro de la cultura organizacional alemana son la persistencia y tenacidad apoyadas por una jerarquía estable, mientras que el tiempo libre no es importante. En cambio, en culturas como la de México, la libertad personal y de expresión, así como el tiempo libre son de gran importancia. Sin embargo, según la dimensión de masculinidad, el tiempo libre no es de gran importancia para México. De tal modo que el capital representa un recurso muy importante.

En Alemania se ejerce una política de reinversión muy estricta enfocada en la visión hacia el futuro. No obstante, en México las organizaciones se enfocan más en los resultados del pasado. En cuanto a las relaciones sociales en el país centroeuropeo (Alemania) se tiene un sentido de vergüenza, pero se asigna prioridad al sentido común y se invierte en personal de por vida. Al contrario, en México el enfoque está en el logro personal y el pensamiento egoísta. Mientras que en Alemania la relación entre superiores e inferiores está basada en la misma aspiración y en salarios equilibrados, en México se muestran distintas aspiraciones entre los líderes y los empleados, y la recompensa se realiza de acuerdo a los resultados. Como se ha mencionado, también hay coincidencia entre los países con una orientación a largo plazo como Alemania y países con una orientación a corto plazo como México: ambas culturas fomentan el trabajo duro y la autoconfianza.

En la dimensión de indulgencia versus restricción, México está posicionado en el segundo lugar con un índice de 97 después de Venezuela, y Alemania se encuentra en medio, en el 52 y 53 lugar, con un índice de 40 de un total de 93 países y regiones que participaron en el WVS. Donde 0 (cero) representa a los países con más restricción y 100 (cien) los países con más indulgencia. 


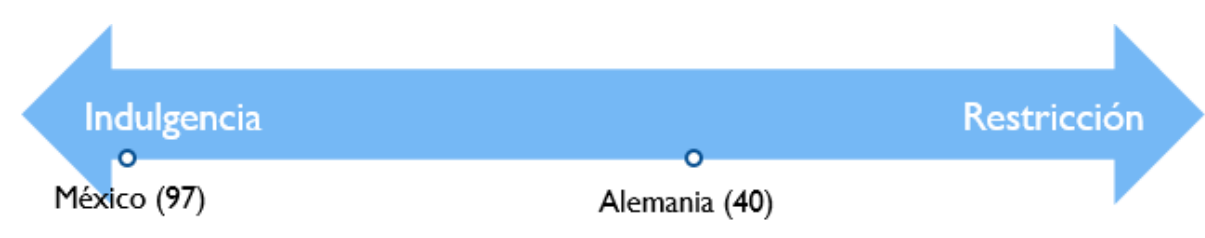

Figura 9. Puntuación de México y Alemania en la dimensión de indulgencia versus restricción

Figure 9. Indulgence vs. restriction scores of Mexico and Germany

Fuente: elaboración propia.

Al igual que en la dimensión anterior, se puede observar una gran brecha entre México y Alemania, lo cual puede originar el surgimiento de conflictos. En general, se pueden observar coincidencias con la dimensión de orientación a largo plazo versus orientación a corto plazo. De acuerdo con su índice en la dimensión de indulgencia, las inversiones a largo plazo y la disciplina moral ocupan una baja prioridad en México; en Alemania tienen una alta prioridad. La percepción de un control de vida dentro de una sociedad, la cual no tiene interconexiones estrechas entre sí, prevalece en México, mientras que a la gente en Alemania se le presenta un sentimiento de desamparo en una sociedad firmemente conectada. Para los individuos en México el tiempo libre es muy importante. Esto también afirma la dimensión de orientación a corto plazo mencionada anteriormente. Para los alemanes el tiempo libre tiene una prioridad baja. La gente en México suele ser más feliz, positiva, extrovertida y optimista que en Alemania, donde las personas son más pesimistas y neuróticas. Reflejado en las organizaciones, esto significa que los empleados alemanes son más serios y están acostumbrados a no expresar sus sentimientos. Los mexicanos dentro del área profesional muestran más una cara feliz independientemente de cómo se sienten.

La Tabla 2 representa un resumen de las diferencias culturales entre México y Alemania, así como de las similitudes al final de la tabla, de acuerdo con la propuesta de Hofstede.
Como se ha mencionado a lo largo de este trabajo, comprender las diferencias y similitudes entre países resulta tarea apremiante en una sociedad cada vez más globalizada. Diversas investigaciones han realizado análisis similares; no obstante, la mayoría de las investigaciones que se han realizado hacen referencia sobre todo a Estados Unidos y países de Asia como China, Corea del Sur o Japón. En dichos trabajos la metodología utilizada es similar a la de las autoras, retomando la definición del modelo de Hofstede y sus dimensiones, así como la comparación de las puntuaciones de los países analizados para de allí poder extraer conclusiones acerca de las diferencias culturales (Díaz, et al., 2016; Leach, 2013; Rodríguez \& Brown, 2014; Villatoro, Chang \& Lane, 2014).

Por lo anterior, se considera relevante el estudio, dado el aumento de las relaciones comerciales que se ha dado en los últimos años entre México y Alemania.

\section{CONCLUSIONES}

No es posible hablar de fortalezas o debilidades de las culturas, ya que cada una de ellas opera de acuerdo con su historicidad y a su sistema de valores y significados. Por tal razón, es importante que las empresas realicen consideraciones culturales dentro de su planeación, sus formas de operar y su liderazgo, sobre todo cuando estas son multinacionales. 
A través de la cultura, las personas tienen un sentimiento de identidad. El comportamiento, la moral y la productividad son elementos esenciales los cuales afectan la cultura. Los valores y patrones que tienen impacto en las acciones de una organización están influenciados por la cultura. En resumen, la cultura se puede considerar como la fuerza motriz para el comportamiento humano. No obstante, la cultura puede ser también causa para la desintegración y el conflicto.

Tabla 2. Similitudes y diferencias culturales entre México y Alemania

Table 2. Cultural similarities and differences between Mexico and Germany

\begin{tabular}{|c|c|}
\hline México & Alemania \\
\hline \multicolumn{2}{|c|}{ Diferencias } \\
\hline Pensamiento político & Pensamiento estratégico \\
\hline Planificación personal y control & Sistemas impersonales \\
\hline No existe confianza en los seguidores & Confianza en los seguidores \\
\hline Gestión no participativa & Gestión participativa \\
\hline Distancia grande entre empleados y líderes & Distancia pequeña entre empleados y líderes \\
\hline $\begin{array}{l}\text { Los subordinados no contactan o contradicen sus líderes } \\
\text { de manera directa }\end{array}$ & $\begin{array}{l}\text { Los subordinados se acercan y contradicen fácilmente con } \\
\text { sus jefes }\end{array}$ \\
\hline Sistema de contabilidad sin gran impacto & Sistema de contabilidad indispensable \\
\hline $\begin{array}{l}\text { Relación entre empleados y organización basada en la } \\
\text { moral }\end{array}$ & $\begin{array}{l}\text { Relación entre empleados y organización basada en los } \\
\text { intereses propios }\end{array}$ \\
\hline Necesidad de figuras poderosas & No existe necesidad de figuras poderosas \\
\hline Libertad personal y de expresión & $\begin{array}{l}\text { Persistencia y tenacidad apoyadas por una jerarquía } \\
\text { estable }\end{array}$ \\
\hline Tiempo libre es muy importante & Tiempo libre no tiene un papel importante \\
\hline Enfoque en los resultados del pasado & $\begin{array}{l}\text { Política de reinversión enfocada en la visión hacia el } \\
\text { futuro }\end{array}$ \\
\hline Logro personal y pensamiento egoísta & Sentido común e inversión en personal de por vida \\
\hline Distintas aspiraciones entre líderes y empleados & Misma aspiración entre superiores e inferiores \\
\hline Recompensa de acuerdo a los resultados & Salarios equilibrados \\
\hline $\begin{array}{l}\text { Baja prioridad de inversiones a largo plazo y la disciplina } \\
\text { moral }\end{array}$ & $\begin{array}{l}\text { Alta prioridad de inversiones a largo plazo y la disciplina } \\
\text { moral }\end{array}$ \\
\hline $\begin{array}{l}\text { Control de vida dentro de una sociedad la cual no tiene } \\
\text { conexiones estrechas entre sí }\end{array}$ & $\begin{array}{l}\text { Sentimiento de desamparo en una sociedad firmemente } \\
\text { conectada }\end{array}$ \\
\hline Gente feliz, positiva, extrovertida y optimista & Gente pesimista y neurótica \\
\hline $\begin{array}{l}\text { Cara feliz en el área profesional independientemente de } \\
\text { cómo se sienten }\end{array}$ & $\begin{array}{l}\text { Empleados serios y acostumbrados a no expresar sus } \\
\text { sentimientos }\end{array}$ \\
\hline \multicolumn{2}{|c|}{ Similitudes } \\
\hline \multicolumn{2}{|c|}{ Planificación en detalle } \\
\hline \multicolumn{2}{|c|}{ Responsabilidad de la planificación de los especialistas } \\
\hline \multicolumn{2}{|c|}{ Retroalimentación a corto plazo } \\
\hline \multicolumn{2}{|c|}{ Enfoque en resultados } \\
\hline \multicolumn{2}{|c|}{ Recompensa según la equidad } \\
\hline \multicolumn{2}{|c|}{ Valores importantes: asertividad, ambición y competencia } \\
\hline \multicolumn{2}{|c|}{ Trabajo duro } \\
\hline \multicolumn{2}{|c|}{ Autoconfianza } \\
\hline
\end{tabular}

Fuente: elaboración propia con base en Hofstede and Minkov, 2010. 
El liderazgo juega un papel muy importante en este contexto. Los líderes están en contacto directo con empleados de culturas distintas. Ellos tienen que ser capaces de influir de manera positiva en el comportamiento, las personalidades y la motivación de sus seguidores, sin importar sus diferencias culturales. El primer paso para poder ofrecer una gestión exitosa de dichas diferencias culturales en el caso de Alemania y México es incrementar la conciencia cultural. Para tal fin, se tiene que entender el concepto de cultura y sus características (Moran, Harris \& Moran, 2011).

Como se ha demostrado en el presente trabajo, el modelo cultural de Hofstede es un valioso punto de partida para explicar las diferencias entre culturas. Se mostraron las seis diferentes dimensiones, en las cuales la cultura puede ser diferenciada según Hofstede, cada una de las cuales tiene influencia en las organizaciones transculturales.

En este trabajo se han considerado también las críticas acerca de los estudios de Hofstede (Blodgett, Bakir \& Rose, 2008; McSweeney, Brown \& Iliopoulou, 2016; Moulettes, 2007; Orr \& Hauser, 2008), además de que se ha considerado que este no es el único modelo que analiza las diferencias culturales. Respecto a estos últimos podemos mencionar a Inglehart (1997) que distingue dos dimensiones: autoridad tradicional $\mathrm{y}$ autoridad secularracional; y la supervivencia y los valores de bienestar; el modelo de Hall (1989) con sus cuatro dimensiones culturales, las cuales representan componentes de la comunicación y controlan el comportamiento; la teoría de las siete orientaciones de valores culturales los cuales se unen en tres dimensiones de valor cultural de Schwartz (2006); o el estudio GLOBE que describe las variables culturales en el liderazgo y en la organización, así como la eficiencia de ambos (House, et al., 1999).
Una de las críticas al modelo de Hofstede es la que afirma que se trata de un modelo simplista por el hecho de que existen nada más cuatro dimensiones de cultura. No obstante, en la edición más reciente del libro de este autor Cultures and Organizations: Software of the mind, publicado en el año 2010, se agregan las dimensiones de «indulgencia versus restricción» y «orientación a largo plazo versus orientación a corto plazo», dimensiones basadas en los estudios del WVS de Misho Minkov para la obtención de una especificación más amplia de la cultura. Por otro lado, se juzga como incorrecto que las dimensiones culturales clasifican a todas las personas de un país de la misma manera. El hecho de que los individuos vivan en el mismo país, no quiere decir que no existen diferencias importantes entre regiones diversas dentro de ese país.

Otro punto que fue muy criticado surge de las mediciones del estudio, las cuales se basan en la empresa IBM en una sola organización con una cultura organizacional particular. En especial, se critica que los empleados de IBM no son representativos para toda la población de un país. Además de lo anterior, como se ha mencionado anteriormente, los estudios de Hofstede iniciaron a finales de los años sesenta del siglo XX, por lo cual no son recientes, y no se han vuelto a repetir a lo largo del tiempo (Garza, Guzmán \& Hernández, 2009).

No obstante, lo anterior, hoy en día existe una genuina preocupación acerca de la estabilidad de las dimensiones culturales de Hofstede y las puntuaciones de los países. Basado en la pregunta de si los datos recogidos por Hofstede hace medio siglo todavía son válidos, recientemente Beugelsdijk, Maseland y Hoorn (2015) realizaron un estudio para identificar la estabilidad de los estudios de Hofstede en el mundo de hoy. El trabajo de los tres autores reveló que, a través del uso de datos de valores contemporáneos, las dimensiones de Hofstede se pueden replicar para cohortes distintos; que 
las puntuaciones en las dimensiones replicadas han cambiado a lo largo del tiempo, sobre todo se pudo observar un cambio en tres dimensiones "colectivismo versus individualismo», «distancia de poder e indulgencia versus restricción»; y que las variaciones se realizaron en la misma dirección, de tal manera que la diferencia de los valores entre los países se mantiene más o menos igual. En consecuencia, la distancia cultural entre los países se mantiene en un nivel relativamente estable.

Los descubrimientos de Beugelsdijk, Maseland y Hoorn (2015) coinciden con las teorías de la modernización, las cuales predicen que en cuanto la riqueza de un país aumenta, los valores de estas sociedades como consecuencia también cambian. El estudio lo demuestra con los valores cambiantes en las dimensiones mencionadas en el párrafo anterior. En resumen, puede destacarse que los cambios culturales en los últimos 50 años son relativos comparando las puntuaciones de los países. Con otras palabras, aunque las puntuaciones en las dimensiones han cambiado a lo largo del tiempo, las posiciones relativas de los países entre sí no se han modificado. De aquí resulta una estabilidad general de las diferencias culturales entre sociedades.

De acuerdo con lo visto anteriormente, y aunque existen críticas acerca del modelo cultural de Geert Hofstede, se considera que este modelo constituye la base para muchos estudios en relación con la comparación entre culturas (Garza, Guzmán \& Hernández, 2009), para el caso que nos ocupa en este trabajo, resulta orientador para comprender las diferencias culturales entre Alemania y México, lo cual resulta importante, considerando que éste último se ubica como el principal socio económico para Alemania en América Latina y El Caribe.
Las limitaciones de este trabajo corresponden a la falta de datos empíricos que validen las conclusiones respecto a las diferencias culturales de los dos países analizados, no obstante, como línea futura de investigación, se realizará una investigación aplicada en la cual sea posible corroborar los hallazgos.

Además, se espera complementar el modelo cultural de Hofstede con otros modelos culturales como los anteriormente mencionados (Inglehart, Hall, Schwartz \& el estudio GLOBE), con la finalidad de comparar y crear una vista más amplia de las similitudes y diferencias culturales entre ambos países.

\section{REFERENCIAS}

Barkley, D. L., \& Eggertsson, M. (2017). Using Hofstede's Model to Improve Multicultural Management in the European Union. Journal of International Management Studies, 17(1), 21-34.

Beugelsdijk, S., Maseland, R., \& Hoorn, A. (2015). Are Scores on Hofstede's Dimensions of National Culture Stable over Time? A Cohort Analysis. Global Strategy Journal, 5(3), 223-240.

Blodgett, F. G., Bakir, A., \& Rose, G. M. (2008). A test of the validity of Hofstede's cultural framework. Journal of Consumer Marketing, 25(6), 339-349.

Díaz, C., Roosevelt, N., Morin, J., Denn, A., Tien, H., \& Steinberg, H. (2016). Customer Service y Hofstede's Cultural Dimensions in China, Ireland, Mexico y the USA. Proceedings of the Academy for Studies in International Business, 16(1), 5-9.

Embajada de México en Alemania. (2016). Relaciones comerciales y de inversión entre México y Alemania. Recuperado de 
https://embamex.sre.gob.mx/alemania/i ndex.php/es/component/content/article/ 419

Garza, M. T., Guzmán, E., \& Hernández, D. (2009). Consideraciones culturales y personales en torno a las negociaciones internacionales. Globalization, Competitiveness y Governability, 3(3), 64-89.

Hall, E. T. (1989). El lenguaje silencioso. Madrid: Alianza Editorial.

Hofstede, G. (1983). Culture's Consequences: International Differences in Work-Related Values. Administrative Science Quarterly, 28(4), 625-629.

Hofstede, G. (1984). Culture's Consequences: International Differences in Work-Related Values. USA: Sage.

Hofstede, G. (1993). Cultures and Organizations: Software of the Mind. Administrative Science Quarterly, 38(1), 132-134.

Hofstede, G., Hofstede, G. J. \& Minkov, M. (2010). Cultures and Organizations: Software of the mind: intercultural cooperation and its importance for survival. London: McGraw-Hill.

House, R. J., Hanges, P. J., Ruiz-Quintanilla, S. A., Dorfman, P. W., Javidan, M., Dickson, M., Gupta, V., \& Koopman, P.L (1999). Cultural Influences on Leadership and Organizations: Project GLOBE. Advances in Global Leadership, 1(2), 171-233.

Inglehart, R. (1997). Modernization and Postmodernization. New Jersey: Princeton University Press.

Khlif, H. (2016). Hofstede's cultural dimensions in accounting research: a review. Meditari Accountancy Research, 24(4), 545-573.
Kirkman, B. L., Lowe, K. B., \& Gibson, C. B. (2006). A quarter century of Culture's Consequences: a review of empirical research incorporating Hofstede's cultural values framework. Journal of International Business Studies, 285-320.

Leach, M. A. (2013). Moderating Effect of Hofstede's Cultural Values on the Locus of Control/Job Performance Relationship of Managers in USA, Mexico, South Korea and Hong Kong. Journal of Business Strategies, 30(1), 15-45.

McSweeney, B., Brown, D., \& Iliopoulou, S. (2016). Claiming too much, delivering too little: testing some of Hofstede's generalizations. Irish Journal of Management, 35(1), 34-57.

Moran, R. T., Harris, P. R., \& Moran, S. V. (2011). Managing Cultural Differences. Oxford: Elsevier Inc.

Moulettes, A. (2007). The absence of women's voices in Hofstede's Cultural Consequences: A postcolonial reading. Women in Management Review, 22(6), 443-455.

Ng, S. I., Lee, J. A., \& Soutar, G. N. (2007). Are Hofstede's and Schwartz's value frameworks congruent? International Marketing Review, 24(2), 164-180.

Orr, L. M., \& Hauser, W. J. (2008). A re-inquiry of Hofstede's cultural dimensions. Marketing Management Journal, 18(2), 119.

Peña, E. (2016). Partner mit gemeinsamen Werten und Prinzipien. Recuperado de http://www.tagesspiegel.de/politik/mexi ko-deutschland-jahr-partner-mitgemeinsamen-werten-undprinzipien/13427622.html 
Rodríguez, A. and Brown, A. (2014). Cultural Differences: A Cross-cultural Study of Urban Planners from Japan, Mexico, the U.S., Serbia-Montenegro, Russia, and South Korea. Public Organization Review, 14(1), 35-50.

Schwartz, S. H. (2006). A Theory of Cultural Value Orientations: Explication and Applications. Comparative Sociology, 5(23), 137-182.
Venaik, S., Zhu, Y., \& Brewer, P. (2013). Looking into the future: Hofstede long term orientation versus GLOBE future orientation. Cross Cultural Management: An International Journal, 20(3), 361-385.

Villatoro, J., Chang, J. \& Lane, S. (2014). Research of ethics, values and crosscultural differences on China, Mexico or the United States. Journal of Technology Management in China, 9(2), 133-154. 
